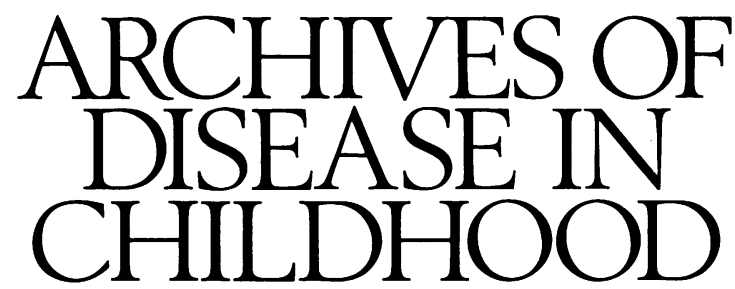

The fournal of the British Paediatric Association

\title{
Monitoring TSH concentrations during treatment for congenital hypothyroidism
}

Treatment of a child with congenital hypothyroidism should be one of the most straightforward and rewarding experiences in paediatrics. As a result of neonatal screening and early diagnosis the prognosis for mental development is greatly improved, treatment itself is simple and inexpensive, and biochemical tests to monitor therapy are widely available. However, most paediaticians will have encountered cases during treatment in which the laboratory results are difficult to interpret as the plasma thyroid stimulating hormone (TSH) concentration is abnormal even though plasma thyroxine-measured either as total thyroxine or free thyroxine-is normal. Such cases are relatively common, particularly during early infancy. For example, $34 \%$ of the children with congenital hypothyroidism reported by Hulse $e t$ al had raised TSH concentrations at the age of 1 year. ${ }^{1}$ Similarly, Abusrewil et al found TSH values above 10 $\mathrm{mU} / \mathrm{l}$ in $38 \%$ of cases tested between 5 and 11 months, ${ }^{2}$ and Germak and Foley found high TSH concentrations at 1 year in $23 \%$ of their patients with thyroid dysgenesis ${ }^{3}$; interestingly, their cases with dyshormonogenesis all had normal TSH values. ${ }^{3}$

Are these discrepancies between TSH and thyroxine concentrations important and what do they mean?

Measurement of thyroxine and TSH reflect slightly different aspects of thyroid status, the former indicating thyroid activity (or replacement treatment with thyroxine), and the latter gives information on the biological effect of circulating thyroid hormone. The reciprocal relationship between the two is well known but the mechanisms behind it are still incompletely understood. However, it seems clear that the set point which defines the concentration of plasma thyroxine at which TSH secretion is normal varies between individual subjects and also changes with age. This set point is associated with high plasma thyroxine values during the first months of life, followed by a progressive decline during childhood. ${ }^{4}$ Change in the set point also occurs as a result of illness, particularly thyroid disease; for example, TSH secretion remains suppressed for some time after hyperthyroidism has been relieved. ${ }^{5}$ The variation in set point during early infancy and delay in establishing a new threshold when plasma thyroxine returns to normal probably account for most of the discrepancies between plasma thyroxine and TSH results encountered during treatment of congenital hypothyroidism.

It has long been known that in adults with hypothyroidism plasma thyroxine must be maintained in the upper nor- mal range to suppress $\mathrm{TSH}^{\circ}{ }^{\circ}$ This is also the case in congenital hypothyroidism, ${ }^{4}$ and many studies have shown that raised plasma TSH concentrations during treatment are almost invariably associated with plasma thyroxine concentrations in the mid to lower part of the normal range. ${ }^{1278}$ In a few cases, raised TSH concentrations have been associated with thyroxine concentrations well above normal and this has been attributed to delay in maturation of the pituitary-thyroid axis. ${ }^{9}$ The children with raised TSH concentrations have generally been receiving a relatively low dose of thyroxine, and the optimum dose which will keep TSH within normal has been estimated at 100-105 $\mu \mathrm{g} /$ $\mathrm{m}^{2},{ }^{10}$ with a high dose relative to body weight $(8$ to $14 \mu \mathrm{g} /$ $\mathrm{kg}$ ) in early infancy. ${ }^{13}$

Delay in reaching a new set point after a change in plasma thyroxine concentration can also result in discrepancies between plasma thyroxine and TSH. This is commonly seen during the first weeks of treatment when plasma thyroxine returns to normal much more quickly than $\mathrm{TSH} .{ }^{3}$ Delay in reaching a new threshold may also cause discrepant results if compliance with treatment is poor. Regular administration of thyroxine shortly before an outpatient visit will largely correct plasma thyroxine without completely suppressing TSH.

Does it matter if plasma TSH is mildly raised during treatment if the patient appears euthyroid with normal growth and normal mental development? In many cases the answer is probably 'no', particularly if there has been a recent change in dosage of thyroxine or if the plasma thyroxine concentration is close to the upper limit of normal. However, we still do not know whether slight undertreatment has any long term adverse effects, for example on psychological outcome, and ideally plasma TSH should be maintained between $0 \cdot 1$ and $5 \mathrm{mU} / 1 .{ }^{3}$ My own practice is to try to keep plasma TSH as near normal as possible during early infancy but to discount slightly raised values in older children, particularly if growth is normal.

Is there a risk of overdosage if treatment aims to keep TSH within the normal range? The answer to this question must be 'yes' until the new immunoradiometric (IRMA)or immunofluorometric (IFMA)-assays for TSH are more widely available. These assays are many times more sensitive than the original radioimmunoassays and can identify TSH concentrations that fall below the lower limits of normal. ${ }^{11}$ There is some evidence that clinical problems such as poor concentration or restlessness may be associated 
with relatively minor degrees of overtreatment ${ }^{8}$ and the new assay methodology will allow further evaluation of this aspect of congenital hypothyroidism.

D B GRANT

Hospital for Sick Children,

Great Ormond Street,

London WCIN $3 \mathcal{F H}$

1 Hulse JA, Grant DB, Jackson D, Clayton BE. Growth, development, and reassessment of hypothyroid infants diagnosed by screening. $B M \mathcal{F} 1982$; 284:1435-7.

2 Abusrewil SSA, Tyfield L, Savage DCL. Serum thyroxine and thyroid stimulating hormone concentrations after treatment of congenital hypothyroidism. Arch Dis Child 1988;63:1368-71

3 Germak JA, Foley TP. Longitudinal assessment of 1-thyroxine therapy for congenital hypothyroidism. $\mathcal{f}$ Pediatr 1990;117:211-9.
4 Sato T, Suzuki Y, Taketani T, Ishiguru K, Nakajima H. Age related change in pituitary threshold for TSH release during thyroxine replacement therapy for cretinism. F Clin Endocrinol Metab 1977;44:553-9.

5 Toft AD, Irvine WJ, Hunter WM, Ratcliffe JG, Seth J. Anomalous plasma TSH levels in patients developing hypothyroidism in the early months after I131 therapy for thyrotoxicosis. F Clin Endocrinol Metab 1974;39:607-9. 6 Reichlin S, Utiger RD. Regulation of the pituitary-thyroid axis in man: relationship of TSH concentration and concentration of free and total thyroxine in plasma. $\mathcal{F}$ Clin Endocrinol Metab 1967;27:251-5.

7 Rezvani I, DiGeorge AM. Reassessment of the dialy dose of oral thyroxine for replacement therapy in hypothyroid children. F Pediatr 1977;90:291-7.

8 Frost GJ, Parkin JM. Management of patients with congenital hypothyroidrost GJ, Parkin JM. Managem.
ism. BMF 1985;290:1485-9.

9 Schultz RM, Glassman MS, MacGillvray MH. Elevated threshold for thyrotropin suppression in congenital hypothyroidism. Am $\mathcal{F}$ Dis Child 1980;134: 19-20.

10 Hodges S, O'Malley BP, Northover BN, Woods KL, Swift PGF. Reappraisal of thyroxine treatment in primary hypothyroidism. Arch Dis Child 1990;65: $1129-32$.

11 Nicoloff JT, Spencer CA. The use and misuse of sensitive thyrotropin assays. f Clin Endocrinol Metab 1990;71:553-8.
Congenital diaphragmatic hernia $(\mathrm{CDH})$ is a rare anomaly occurring in one in $\mathbf{3 6 0 0}$ live births. If affected fetuses are taken into account the incidence is increased to one in $2200 .^{1}$ An increasing proportion of these cases are being diagnosed as a result of routine antenatal scanning. There remain a significant number of affected patients who present for the first time in the neonatal period and a further group presenting as older infants or children.

\section{Neonatal diagnosis and pitfalls}

The classical presentation of a neonate in respiratory distress, with a scaphoid abdomen, apparent dextrocardia, and multiple air and fluid filled bowel loops in the left hemithorax on chest radiography presents no diagnostic difficulty. Occasionally the chest radiograph is taken before the passage of swallowed air into the herniated intestine and will thus demonstrate a radio-opaque hemithorax. The absence of intestinal gas in the abdomen should provide the diagnostic clue in these cases. ${ }^{2}$ In all cases the abdomen should be included on the radiograph if $\mathrm{CDH}$ is suspected. The existence of a previously normal chest radiograph does not preclude the diagnosis. ${ }^{3}$ The defect in the diaphragm may be plugged by a solid abdominal viscus, for example, liver, spleen, or omentum. ${ }^{4}$ The delayed appearance may on some occasions be related to initially raised intrathoracic pressure which when lowered allows the abdominal viscera to enter into the affected hemithorax. ${ }^{5}$

There is a further group of neonates with delayed appearance of right diaphragmatic hernia associated with group B streptococcal infection, and neonates with relapsing streptococcal infection should have this diagnosis positively excluded. ${ }^{6}{ }^{7}$ Right sided hernias may present as a pleural effusion, the obstructed hepatic venous outflow from herniated liver causing vascular congestion and transudation from the liver surface. ${ }^{4}$ The effusion may compress the adjacent lung resulting in atelectasis which may mimic primary pulmonary pathology, or may prevent identification of either the herniated liver or the diaphragm itself. Ultrasound should establish the diagnosis in these cases. Paraoesophageal and oesophageal hiatal hernias are generally associated with vomiting rather than respiratory embarrassment. Large paraoesophageal hernias are usually identified in the posterior right cardiophrenic region and easily confirmed on barium studies. The differentiation of cystic adenomatoid malformation, congenital lobar emphy- sema, and CDH may present diagnostic difficulty as they may all present with respiratory distress at or shortly after birth. $^{9-11}$ If the chest radiograph is taken too early the affected lung in cystic adenomatoid malformation and congenital lobar emphysema may be fluid filled. There are several diagnostic pointers which assist in establishing the correct diagnosis. The presence of normal intestinal gas shadows within the abdominal cavity should raise doubts about the diagnosis of CDH. The position of the tip of the nasogastric tube may be helpful when the stomach lies above the diaphragm, and in these instances it may be a prognostic indicator, as the presence of an intrathoracic stomach has been equated with an adverse prognosis. ${ }^{12}$ On rare occasions air or contrast may be instilled via the nasogastric tube to determine the position of the stomach and small intestine. Ultrasound may prove helpful and may demonstrate the diaphragmatic defect with bowel or, if on the right, liver passing through. Congenital lobar emphysema usually involves the upper lobes and the chest radiograph most commonly shows hyperlucency rather than an appearance similar to bowel gas shadows. 9 There have been reports of the other congenital bronchopulmonary malformations being misdiagnosed as $\mathrm{CDH}$, but these reports stress the importance of careful interpretation of the chest and abdominal radiographs, and if necessary, the use of ultrasound. ${ }^{13} 14$

Eventration is more common on the right, usually contains liver, and is most easily diagnosed by ultrasound. Occasionally the fine distinction between a partition containing a few strands of muscle, that is, a diaphragmatic eventration, and a peritoneal sac without striated muscle, that is, a hernia, may prove difficult even at surgery, but in such cases the distinction is unimportant. ${ }^{15}$

Staphylococcal pneumonia that has progressed to produce pneumatocoeles may have an appearance on chest radiography that on first inspection mimics $\mathrm{CDH} .{ }^{15}$ Staphylococcal pneumonia may affect either lung and the bowel gas pattern will be normal. The clinical presentation will be different and the presence of earlier films should prove helpful.

Urgent surgery for $\mathrm{CDH}$ has been superceded by a period of preoperative resuscitation and stabilisation which may extend over several days. ${ }^{16}$ The rapid presumptive diagnosis of $\mathrm{CDH}$ remains essential for the correct management to be instituted. The period of preoperative stabilisation allows ample time for the definitive diagnosis to be established by the full range of necessary radiological investigations if required. 